MATEC Web of Conferences 32, 04006 (2015)

DOI: $10.1051 /$ matecconf $/ 20153204006$

(C) Owned by the authors, published by EDP Sciences, 2015

\title{
6DOF optical tracking system using afocal optics for image guided surgery
}

\author{
You Seong Chae ${ }^{1}$, Seung Hyun Lee ${ }^{1}$, Hyun Ki Lee ${ }^{2}$, Hyungsuck Cho ${ }^{3}$, and Min Young Kim ${ }^{1,4}$ \\ ${ }^{1}$ School of Electrical Engineering and Computer Science, Kyungpook National University,80 Daehakro, Buk-gu, Daegu 41566, \\ Korea (Tel : +82-53-950-7233; E-mail: mykim@ee.knu.ac.kr) \\ ${ }^{2}$ Medical Robot Unit, Kohyoung Technology Ltd. 15F Halla Sigma Valley, 345-90 Gasan-Dong, Geumcheon-Gu, Seoul, Korea \\ ${ }^{3}$ Department of mechanical Engineering, Korea Advanced Institute of Science and Technology, 291, Daehak-ro, Yuseong-gu, \\ Daejeon 34141, Korea \\ ${ }^{4}$ Department of Biomedical Engineering, Johns Hopkins University, Baltimore, MD 21205, United States
}

\begin{abstract}
Image guided surgery using medical robots is becoming popular these days. For image guided surgery, a tracking system is required to provide $6 \mathrm{DOF}$ information of patient coordinate, surgical instruments and medical robots used in surgery. To provide $6 \mathrm{DOF}$ information, a marker has to be attached to the target. However, it is hard to use many markers all together because the markers will take too much space in the surgical area. The tracking system proposed in this study utilizes down sized markers compared to traditional markers by using micro-engraved datacoded pattern with a lens instead of using geometrically specified marker spheres as a tracking target. A tracking system is developed that has a measurement area of $1 \mathrm{~m}$ to $2.5 \mathrm{~m}$ from the tracking system. Experiment has been done for surgical navigation using the proposed tracking system and a medical robot.
\end{abstract}

\section{Introduction}

Image guided surgery is becoming popular because of comfort, safety, accuracy and quicker recovery [1]. Optical tracking system, which is used for a tracking system, is used for tracking targets for patient registration, surgical navigation and augmented reality [2-4]. These days image guided surgery is applied to more complicated surgeries. As a result, more markers are required for tracking targets in surgery. However, the size of traditional markers which are made with geometrically specified marker spheres limits the number of target markers used in surgery. In a previous study, we proposed an algorithm that can downsize the marker maintaining the accuracy of the tracking system [5]. In this paper, an improved system using the proposed algorithm of the previous study is shown. The marker proposed in this study is smaller than traditional markers and allows more markers to be used in surgery.

\section{METHOD}

The proposed system utilizes a special marker which has fisheye lens and micro-engraved data coded pattern as components. The position of the marker is measured by stereo vision and the orientation is measured using the data coded pattern.

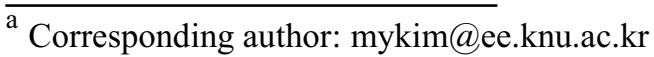

\subsection{Afocal marker}

We named the marker used in the proposed system Afocal marker. The marker has a fisheye lens, microengraved data coded pattern behind the lens and IR light source behind the data coded pattern as shown in Fig. 1.

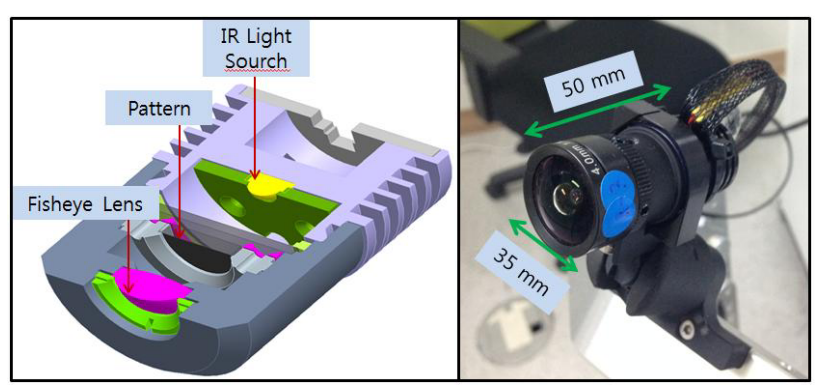

Figure 1. The proposed afocal marker. The components of the marker(left) and the size of the marker(right).

The proposed tracking system utilizes stereo camera. The difference is that the camera of the proposed tracking system is out of focused. When observing afocal marker with focused camera, the marker is shown as a small dot in the camera image. However, when the camera is out of focused, the small dot increases due to bokeh effect. Inside the increased circular bokeh, the micro-engraved

This is an Open Access article distributed under the terms of the Creative Commons Attribution License 4.0, which permits unrestricted use, distribution, and reproduction in any medium, provided the original work is properly cited. 
data coded pattern is shown with magnification [6]. This is shown in Fig. 2.

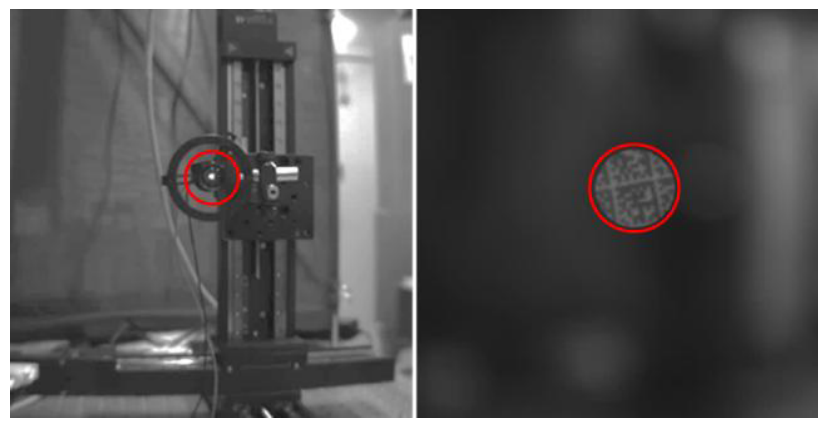

Figure 2. Image observing afocal marker with lens focused(left) and image observing afocal marker with lens out of focused(right).

\subsection{Calibration}

To use stereo vision, calibration is required. However, the proposed system uses out of focused camera lenses and therefore using chessboard for calibration, which is popular, is not capable. Therefore, we use a special calibration board which has retro-reflective ball lenses in a specific arrangement. For the ball lenses are placed in a specific arrangement, each ball lens can be recognized even if the calibration board pose varies. The calibration board and an image observing the calibration board is shown in Fig. 3.

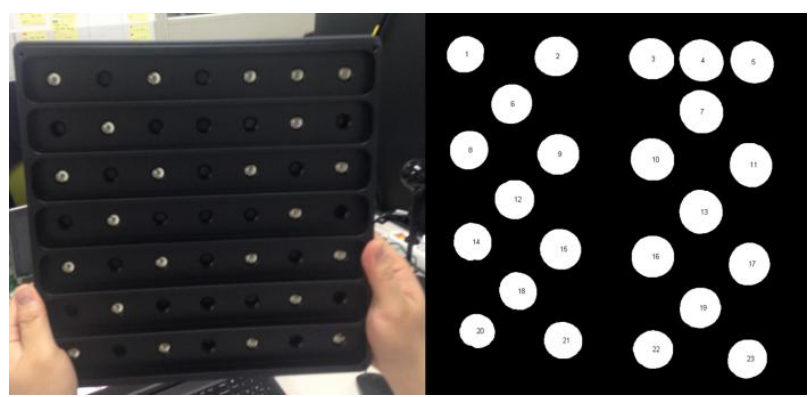

Figure 3. Calibration board(left) and the image observing the calibration board(right).

\subsection{Position measurement}

The position of afocal marker is measured by stereo vision. Before measuring stereo vision, finding the marker in the image and obtaining corresponding pixel points from each camera image is required. As shown in Fig. 2, there are data coded patterns shown in the bokeh of the image. By decoding the pattern, the system can recognize the ID encoded in the pattern. After detecting the corresponding center point of the bokeh that have the same ID encoded pattern, stereo vision is applied and measures the position of the afocal marker.

\subsection{Orientation measurement}

The orientation measurement is done by using a relation between the pixel coordinate of the patterns shown in the image and the corresponding pattern coordinate of the pattern. The datamatrix are engraved on plane glass with accurate positioning and each datamatrix are encoded containing the ID of marker, and the $2 \mathrm{D}$ coordinate of itself. The relation of the pixel coordinate of the datamatrix and the pattern coordinate of the datamatrix is shown in Eq.(1).

$$
\begin{aligned}
& \mathrm{s}\left[\begin{array}{l}
u^{\prime} \\
v^{\prime} \\
1
\end{array}\right]=[A][R][C]\left[\begin{array}{l}
u \\
v \\
1
\end{array}\right] \\
& \left(s=r_{31} u+r_{32} v+r_{33} f_{f}\right) \\
& {[A]=\left[\begin{array}{ccc}
-\frac{f_{c}}{p w} . & 0 & u_{c}^{\prime} \\
0 & -\frac{f_{c}}{p h .} & v_{c}^{\prime} \\
0 & 0 & 1
\end{array}\right]} \\
& {[C]=\left[\begin{array}{ccc}
1 & 0 & -u_{c} \\
0 & 1 & -v_{c} \\
0 & 0 & f_{f}
\end{array}\right]}
\end{aligned}
$$

Here, $u^{\prime}$ and $v^{\prime}$ is the image coordinate of the datamatrix, $u$ and $v$ is the pattern coordinate of the data matrix, $[A]$ is the intrinsic matrix which contains the calibration parameters of the camera, $[C]$ is the intrinsic matrix of the marker lens and $[R]$ is the rotation matrix of the marker. $f_{c}$ is the focal length of the camera lens, $f_{f}$ is the focal length of the fisheye lens, and $p w$ and $p h$ are the pixel width and height, respectively, of the CCD. The orientation measurement is done by calculating the orientation matrix of Eq.(1).

\section{Experiment}

Experiment was performed to verify the usability of the proposed tracking system for image guided surgery. The scenario is positioning a surgical instrument in the right place using a robot and inserting the surgical instrument in the patients head. We used a head phantom, CT image, robot equipped on the bead, surface measurement system and a surgical navigation system to perform the experiment. Markers are attached to the bead, robot and surface measurement device. The experiment setup is shown in Fig.4.

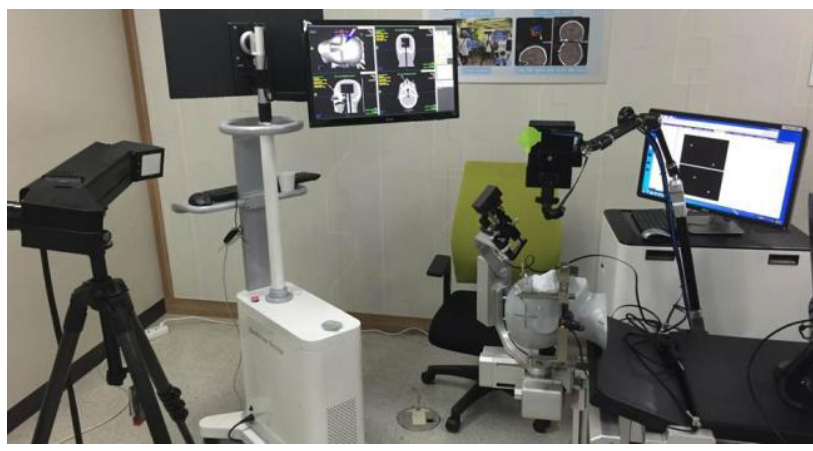

Figure 4. Experimental setup. The tracking system is shown in the left, surgical navigation system with a monitor in the middle, head phantom on the bed at the right with a surface measurement device above it and a robot attached on the bed under the head phantom. 
The marker attached to the bed is for reference coordinate. The surface measurement device measures the surface of the head phantom and using the 6DOF information of the reference marker and the marker fixed to the surface measurement device, registration of the CT data to the head phantom was performed. Then the robot is controlled to position the surgical instrument in the right place using the information of target position information and the 6DOF information of the marker attached to the robot. Surgical navigation system provided real time information of the surgical instrument and CT image. The result of positioning the surgical instrument is shown in Fig. 5.

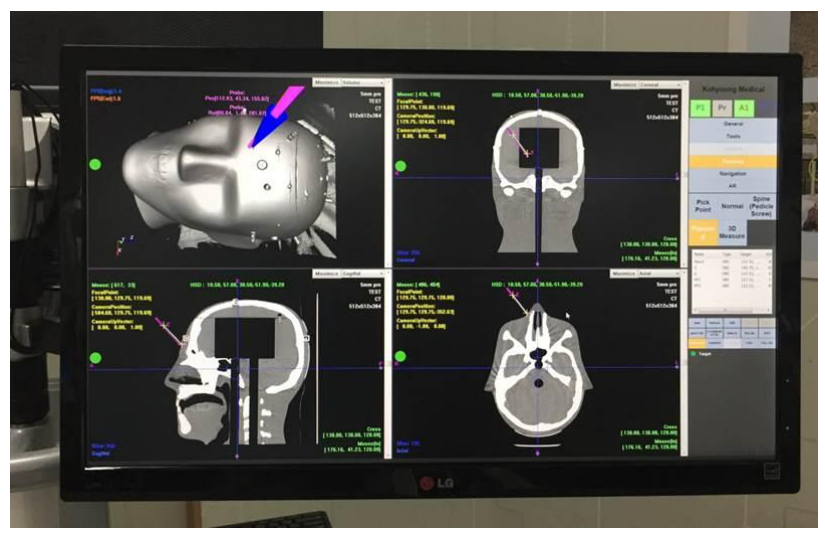

Figure 5. Display monitor of surgical navigation system. The blue cone indicates where the surgical instrument should be position and the pink line is the surgical instrument displayed according to the $6 \mathrm{DOF}$ information of the surgical instrument provided by the proposed optical tracking system.

\section{Discussion}

The experiment showed that the proposed optical tracking system down sizes the target marker and is capable to use in image guided surgery. However there is a problem left to be solved. Fig. 6 shows the datamatrix of the marker observed in the image and the recognition of the datamatrix.

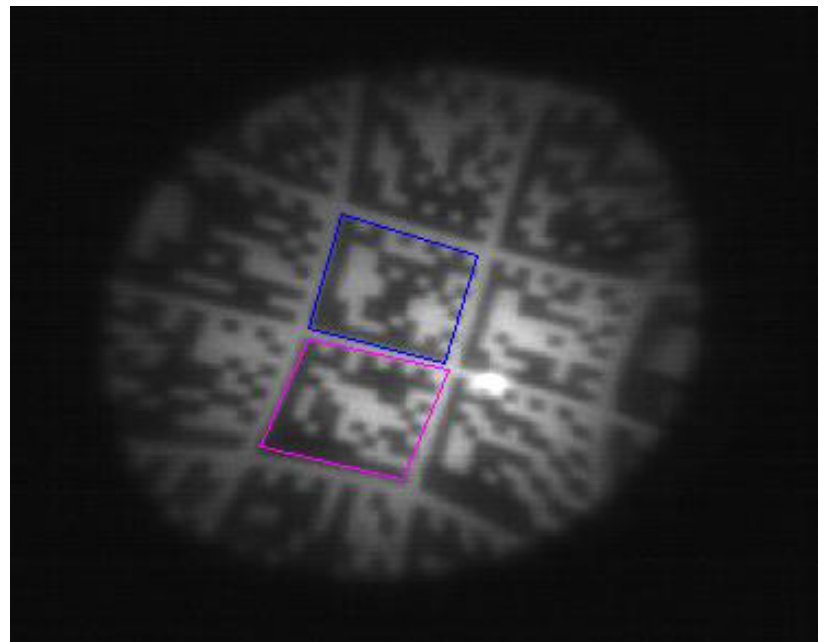

Figure 6. Marker observed in the image. Recognized datamatrix are drawed with colored rectangle. Distortion is observed and the datamatrix that shows less distortion are recognized.
In the image, distortion is observed. Datamatrix that have less distortion are recognized and the decoded information and the pixel coordinate of the datamatrix is used for orientation measurement. However, the distortion will degrade the performance of orientation tracking. Distortion correction will imrpove the accuracy of the tracking system. However, the distortion depends on the camera lens and also the lens of marker. Therefore this problem is difficult to solve.

\section{Conclusion}

In this paper, an optical tracking system which is capable of using smaller sized target markers is proposed. It has an advantage that more markers can be used in image guided surgery. Experiment was performed to show the usability of the proposed tracking system for image guided surgery and the result verified the performance of the tracking system. There are further work to improve the accuracy by image processing and distortion correction. Experiment for accuracy of the tracking system is on progress.

\section{Acknowledgments}

This work is supported by the Technology Innovation Program(10040097) funded by the Ministry of Knowledge Economy(MKE, Korea).

\section{References}

1. W.E.L. Grimson, R. Kikinis, F.A. Jolesz, P.M. Black. Imag-guided surgery. Sci Am, 280.6, 54-61( 1999)

2. G. Eggers, B. Kress, S. Rohde, J. Mühling. Intraoperative computed tomography and automated registration for image-guided cranial surgery. Dentomaxillofacial Radiology. 38.1, 2833(2009)

3. R.R. Fulton, S.R. Meikle, S. Eberl, J. Pfeiffer, C.J. Constable, and M.J. Fulham. Correction for head movements in positron emission tomography using an optical motion-tracking system. Nuclear Science, IEEE Transactions on, 49.1, 116-123 (2002).

4. M. Ribo, A. Pinz, and A.L. Fuhrmann, A new optical tracking system for virtual and augmented reality applications. IMTC, Proceedings of the 18th IEEE. Vol. 3 (2001)

5. Y.S. Chae, S.H. Lee, H.K. Lee, M.Y. Kim, Optical coordinate tracking system using afocal optics for image-guided surgery. IJCARS, 10.2,231-241(2015)

6. A. Mohan, G. Woo, S. Hiura, Q. Smithwick, R. Raskar, Bokode: imperceptible visual tags for camera based interaction from a distance. ACM TOG, 28.3,98(2009) 